
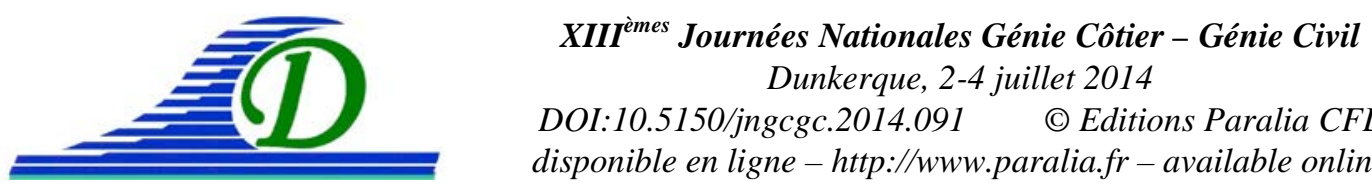

DOI:10.5150/jngcgc.2014.091 @ Editions Paralia CFL

disponible en ligne - http://www.paralia.fr - available online

\title{
Evolution sédimentaire (2010-2014) de la zone d'expérimentation de clapage du Grand Port Maritime de Rouen en baie de Seine orientale
}

\author{
Stella MARMIN ${ }^{1}$, Jean Claude DAUVIN ${ }^{1}$, Patrick LESUEUR ${ }^{1}$, \\ Sandrine SAMSON ${ }^{2}$, Patrice TOURNIER ${ }^{2}$
}

1. Université de Caen Basse-Normandie, Laboratoire Morphodynamique Continentale et Côtière, CNRS UMR CNRS 6143 M2C, 24 rue des Tilleuls, 14000 Caen, France. stella.marmin@unicaen.fr

2. Grand Port Maritime de Rouen, 34 Boulevard de Boisguilbert, BP 4075, 76022 Rouen Cedex 3, France

env@rouen.port.fr ; scan@rouen.port.fr

\section{Résumé :}

Un projet d'expérimentation de clapage de sédiment provenant du dragage d'entretien du chenal d'accès du Grand Port Maritime de Rouen (GPMR) ont été menées en 20122013 sur deux sites voisins de la baie de Seine. Basés sur une approche de type BACI (Before After Control Impact), les changements spatio-temporels des caractéristiques morphologiques et sédimentologiques ont été étudiés au cours de 12 suivis entre avril 2010 et novembre 2013. Les immersions expérimentales ont généré la formation d'un premier dépôt conique de $5 \mathrm{~m}$ de hauteur (MABIO) et un deuxième dépôt plus étalé ayant une hauteur légèrement inférieure à $2 \mathrm{~m}$ (MASED). Une accumulation de sables fins et un envasement ont été observés sur et à proximité des deux zones de clapage avec une teneur en vase atteignant $50 \%$ contre $5 \%$ avant les immersions. Concernant le dépôt conique, un étalement des particules les plus fines a été montré vers le sud ouest. Les vidéos sous-marines effectuées à l'aide d'un mini ROV Seabotix confirment les résultats des analyses granulométriques et levés bathymétriques. Elles mettent en évidence la forte hétérogénéité des fonds principalement aux abords des zones de clapage.

\section{Mots-clés :}

Clapage, Baie de Seine orientale, Granulométrie, Bathymétrie, Vidéos sous-marines.

\section{Introduction}

Dans le contexte international de l'accroissement de la taille des navires, le GPMR se doit de maintenir dans son chenal d'accès une hauteur d'eau suffisante pour permettre la navigation en toute sécurité. Ainsi, 4,5 millions de $\mathrm{m}^{3}$ de sédiments sont dragués chaque année pour l'entretien des fonds de la partie aval de son chenal de navigation. Ces importantes quantités de sédiments sableux fins $(63-200 \mu \mathrm{m})$ faiblement envasés $(0$ $30 \%$ de fines) sont jusqu'alors immergées en mer sur le site du Kannik localisé à 


\section{Thème 6 - Gestion durable des zones littorales et estuariennes}

l'ouverture de la fosse nord de l'estuaire ; on parle alors de clapage. Cependant, ce site actuel des déblais de dragages arrive à saturation en 2014, nécessitant la recherche d'un site alternatif à l'extérieur de l'estuaire. D'autres sites substitutifs ont été étudiés et un projet d'expérimentation de clapage de sédiments a été réalisé dans le secteur admis comme le meilleur compromis : le site de Machu en baie de Seine orientale. L'expérimentation a pour objet de caractériser l'impact de clapages répétés sur l'environnement dans le secteur retenu et rechercher les pratiques minimisant cet impact. Seul le contenu sédimentaire de notre étude biosédimentaire est présenté ici, sachant que l'impact des dépôts sur le benthos fait l'objet d'un important développement (MARMIN, 2013 et publications en cours). Pour l'ensemble de l'étude, des conclusions générales sont difficiles à établir, ce qui rejoint les observations réalisées ailleurs et suggère que les évaluations doivent être effectuées au cas par cas (HARVEY et al., 1998 ; SIMONINI et al., 2005 ; BOLAM et al., 2006).

\section{Matériels et méthodes}

\subsection{Méthodologie : stratégie et plan d'échantillonnage}

Un ensemble de 17 stations a été sélectionné pour suivre l'impact de l'expérimentation de clapage (figure 1). Trois radiales ont été orientées selon la courantologie et une modélisation mathématique préliminaire (GPMR-ARTELIA). Les sept stations de la radiale AK4 ont été réparties autour du site de clapage MABIO afin de suivre l'impact de dépôt à un rythme saisonnier $\left(4 \times 250000 \mathrm{~m}^{3}\right)$. Les quatre épisodes de clapage ont été réalisés d'avril 2012 à février 2013 sur une zone rectangulaire de 98 ha qui entourent les stations AK4C et AK4D. Le sédiment a été déposé en répartissant le sédiment sur toute la surface rectangulaire prédéfinie. Les sept stations de la radiale AK7 ont été disposées autour du site de clapage MASED afin de suivre l'impact d'un clapage d'un $1 \mathrm{Mm}^{3}$. Un point de clapage unique a été défini afin de former un dépôt conique, ce qui correspond à 7 mois d'activités de clapage réalisés de mai à décembre 2012. La radiale AK8 de référence, hors impact des immersions, comprend trois stations. Elle a été sélectionnée en raison d'une similitude entre la granulométrie du sédiment et sa macrofaune avec les sites des radiales AK7 et AK4 lors des deux suivis menés avant dépôt lors de la recherche d'une zone d'expérimentation de clapage en baie de Seine (JOURDE \& DUBUT, 2009 ; ALIZIER \& DAUVIN, 2009).

\subsection{Traitements et analyses des données}

Les prélèvements de sédiments ont été effectués à la benne Van Veen sur les 17 stations pour l'analyse de la granulométrie par tamisage mécanique. Des cartographies ont été réalisées et illustrent la distribution spatiale des classes granulométriques des stations sur la zone de Machu entre avril 2010 et novembre 2013. Les résultats ont été exprimés en pourcentage du poids total de l'échantillon, regroupés en cinq classes 


\section{XIII ${ }^{\text {èmes }}$ Journées Nationales Génie Côtier - Génie Civil \\ Dunkerque, 2-4 juillet 2014}

granulométriques : graviers et galets $(>2 \mathrm{~mm})$, sables grossiers $(0,5-2 \mathrm{~mm})$, sables moyens $(250-500 \mu \mathrm{m})$, (4) sables fins $(63-250 \mu \mathrm{m})$ et vase (ou fines, $<63 \mu \mathrm{m})$. Ces classes ont été établies selon la classification de WENTWORTH (1922) modifiée par FOLK et WARD (1957) et FOLK (1966); elle est basée sur les normes françaises AFNOR.

Afin de caractériser la morphologie des fonds sur les zones de clapage, l'évolution de la bathymétrie a été étudiée à partir d'un sondeur multi-faisceaux par le GPMR. Les résultats sont présentés à partir de cartes illustrant le différentiel de la bathymétrie des sites d'immersion avant et après la fin des épisodes de clapage et des coupes topographiques des deux sites ont permis de caractériser la morphologie des dépôts. Une série de photographies prises lors des prélèvements de sédiments et des vidéos sous-marines ont été réalisées régulièrement avant et pendant les opérations de clapage afin d'observer l'évolution des fonds sédimentaires au cours du temps.

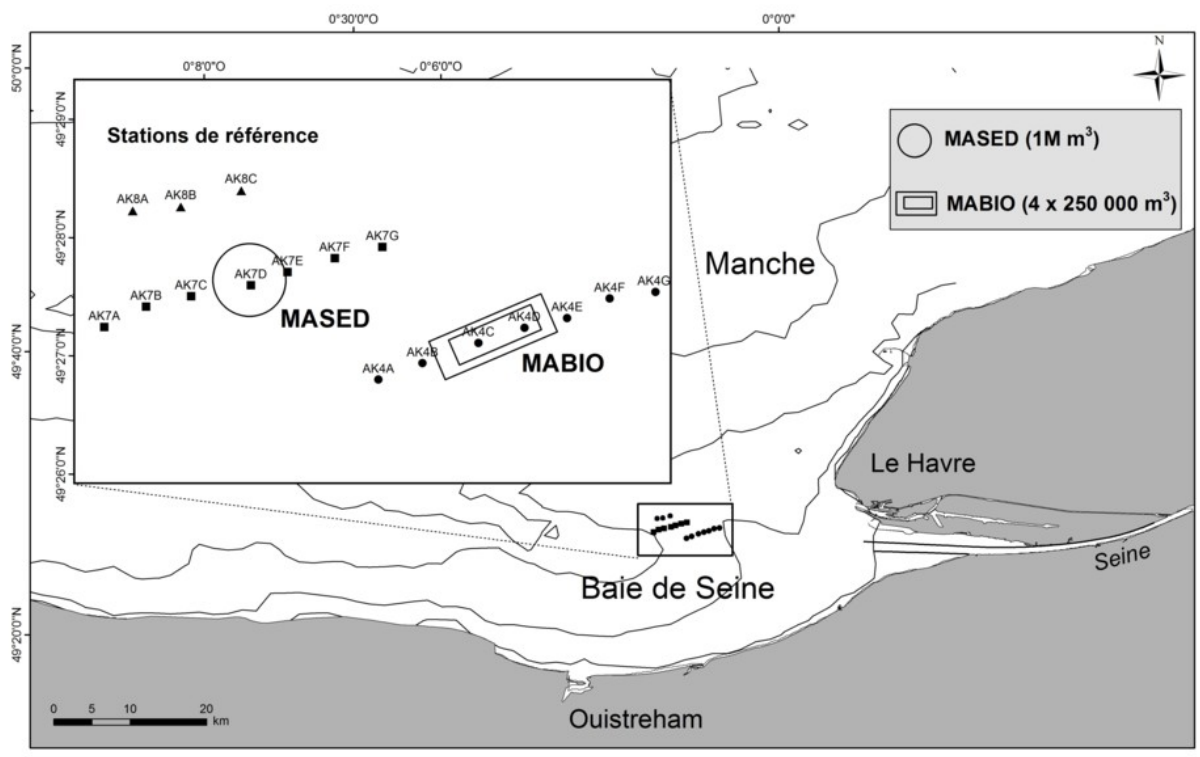

Figure 1. Localisation des 17 stations échantillonnées entre avril 2010 et novembre 2013.

\section{Résultats}

\subsection{Bathymétrie}

L'accumulation constituée sur le site de MASED est un cône de hauteur légèrement inférieure à $5 \mathrm{~m}$ pour un rayon à sa base de l'ordre de $350 \mathrm{~m}$ (figure 2); il est marqué d'une dépression de 1 à $1,5 \mathrm{~m}$ de profondeur avec un rayon de $50 \mathrm{~m}$ à son sommet. Le calcul des évolutions bathymétriques depuis le levé de référence de novembre 2011 met en évidence un apport de $735000 \mathrm{~m}^{3}$ de sédiment, et correspond à un taux de stabilité des sédiments sur leur site d'immersion de $73 \%$. Sur le site de MABIO, le dépôt est 
d'une hauteur légèrement inférieure à $2 \mathrm{~m}$ et d'une longueur approximative de $1200 \mathrm{~m}$ pour une largeur de $700 \mathrm{~m}$.

Le calcul des évolutions bathymétriques depuis le levé de référence de novembre 2011 met en évidence sur ce second site un apport de $590000 \mathrm{~m}^{3}$ de sédiment, correspondant à un taux de stabilité des sédiments sur leur site d'immersion de 59\%.
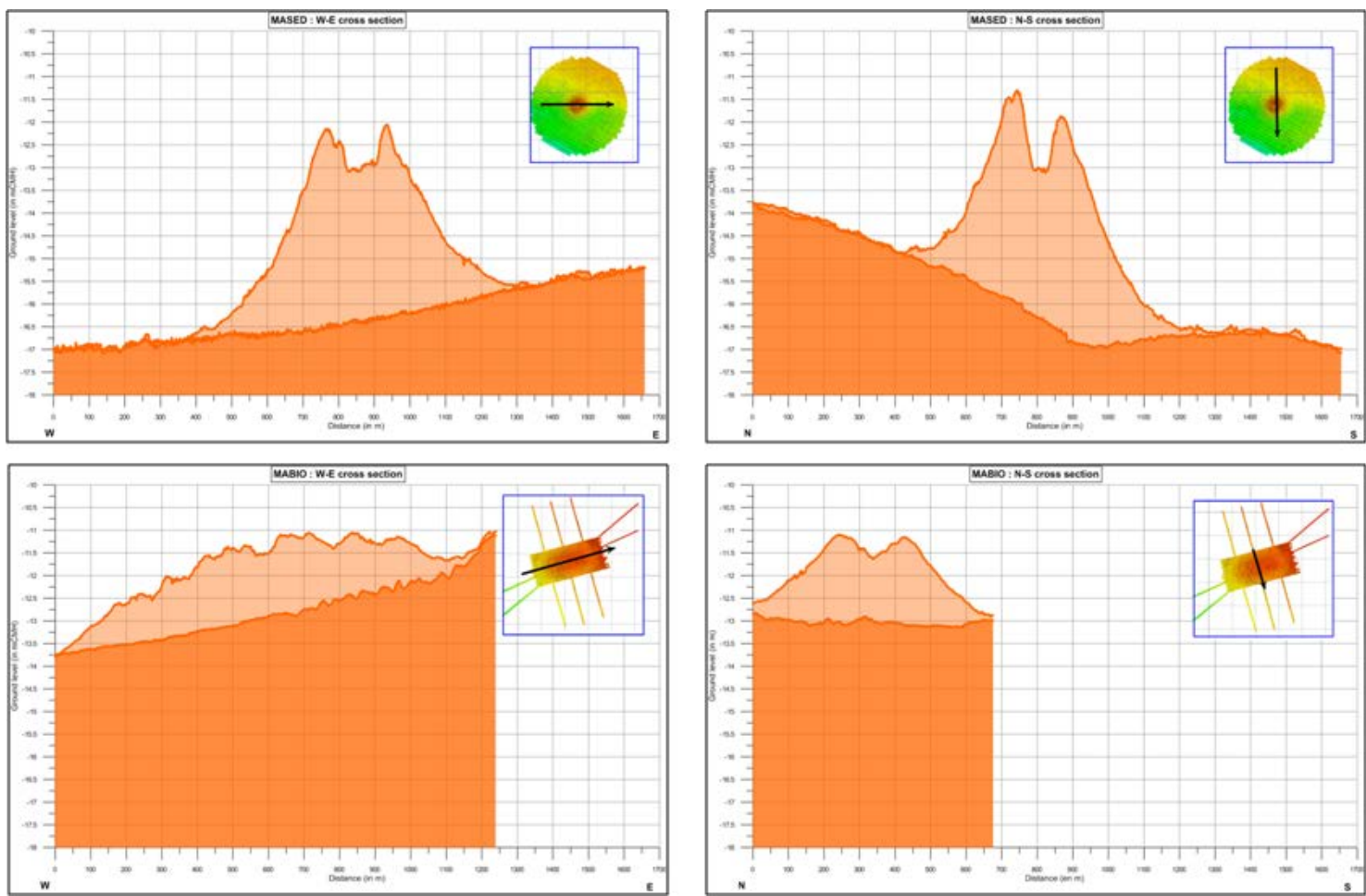

Figure 2. Coupes transversales (W-E) et longitudinales $(N-S)$ des sites d'expérimentation de clapage. Haut : MASED, bas : MABIO (source : GPMR).

\subsection{Granulométrie du sédiment}

La figure 3 présente la répartition spatiale des classes granulométriques des 17 stations entre avril 2010 et novembre 2013.

Avant les clapages (avril 2010 et novembre 2011) une grande dominance des sables est constatée : les fonds des trois stations de la radiale de référence AK8 sont formés de sables moyens et fins. La proportion en vase est toujours inférieure à $1 \%$. Le long des radiales $\mathrm{AK} 4$ et $\mathrm{AK} 7$, la proportion des sables moyens a tendance à être plus élevée, avec une nette variabilité spatiale et temporelle naturelle. Par exemple, la fourchette d'extension maximale de la proportion des sables moyens varie de 31 à $73 \%$ en avril 2010 (respectivement à $\mathrm{AK} 4 \mathrm{~B}$ et $\mathrm{AK} 4 \mathrm{D}$ ) et de 9 à $60 \%$ en novembre 2011 (respectivement à $\mathrm{AK} 4 \mathrm{~A}$ et $\mathrm{AK} 4 \mathrm{E}$ ). La proportion des fines (vase) est restée inférieure à $5 \%$. 


\section{XIII ${ }^{\text {èmes }}$ Journées Nationales Génie Côtier - Génie Civil \\ Dunkerque, 2-4 juillet 2014}
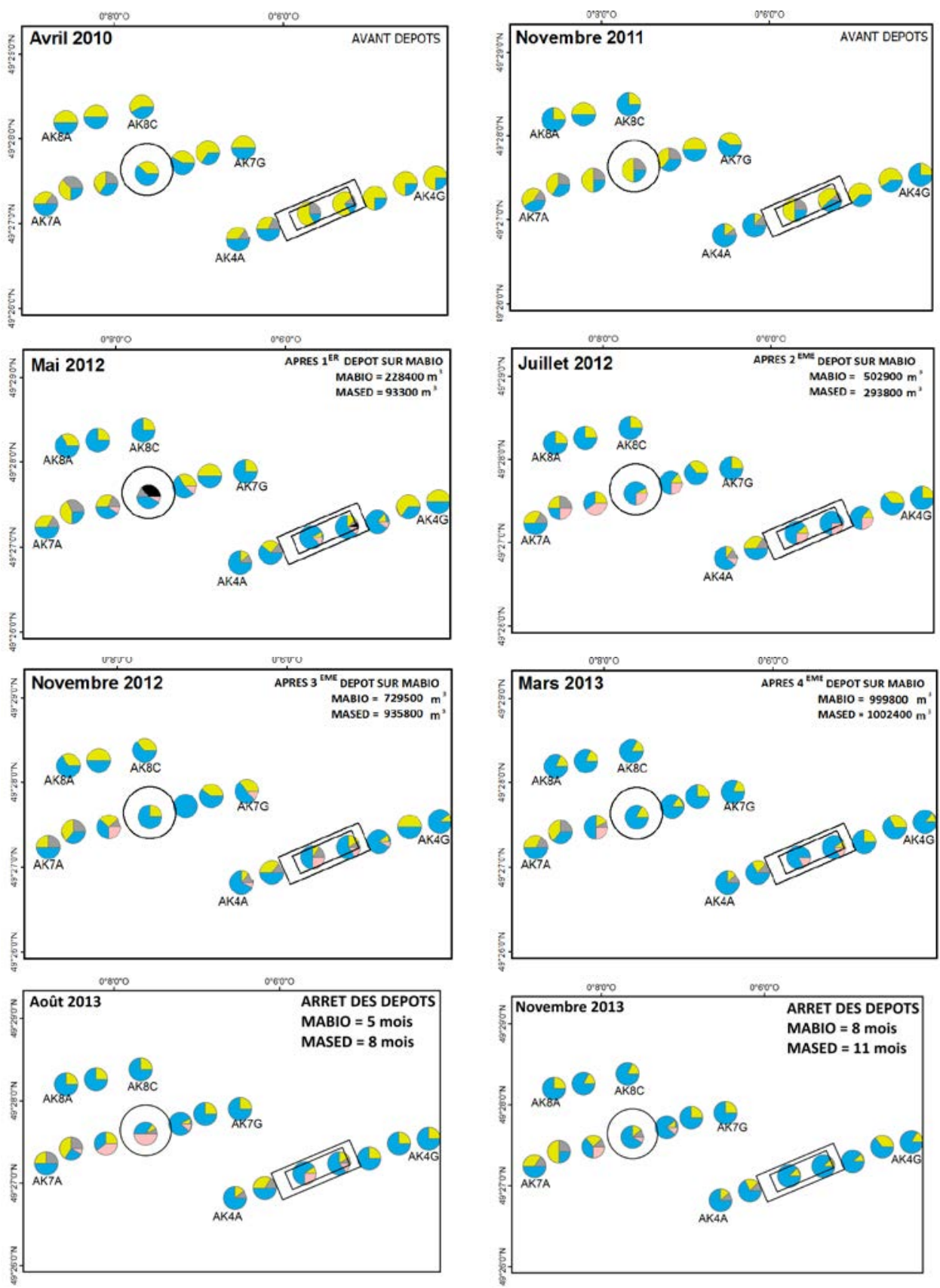

Figure 3. Distribution spatiale des classes granulométriques des 17 stations de la zone de Machu entre mai 2012 et novembre 2013 (en noir = gravier et galet, en gris = sable grossier, en jaune = sable moyen, en bleu = sable fin et en rose $=$ vase $)$.

Durant la période de clapage, entre mai 2012 et novembre 2013, la dominance des sables moyens à fins (modes compris entre 125 et $500 \mu \mathrm{m}$ ) est observée. A noter en mai 2012, une contribution significative de graviers (mode à 31-40 mm) sur les stations de 


\section{Thème 6 - Gestion durable des zones littorales et estuariennes}

clapage AK7D (37\%) et AK4D (7\%) en raison de l'apport de sédiments plus grossiers provenant de l'approfondissement du chenal de navigation du GPMR. Entre juin 2012 et mars 2013, la fraction des graviers a nettement diminué au profit de fractions plus fines (sables moyens à vases) les ayant recouverts. Une importante fraction de fines a été mesurée principalement sur les stations de clapage et leurs stations les plus proches. Sur le site de MABIO, la fraction de fines a augmenté graduellement de mai 2012 à octobre 2012 (jusque 31\% sur AK4C en octobre 2012 ; figure 3) puis diminué progressivement jusqu'à la fin des immersions en novembre 2013. Sur le site de MASED, une plus importante (30\%) fraction vaseuse a été détectée sur la station AK7D jusqu'en juillet 2012, date à laquelle environ $300000 \mathrm{~m}^{3}$ de sédiments avaient été déposés. Durant la suite du suivi, cette fraction s'est réduite à 6\%. En mars 2013, trois mois après la fin des immersions sur le site de MASED, la fraction vaseuse était devenue négligeable ( $1 \%$ sur le site de rejet AK7D). Une tendance inverse a toutefois été constatée sur les stations les plus proches du lieu de clapage avec, par exemple, 53\% de fines sur AK7C en janvier 2013, suggérant l'étalement des fines vers le SW.

La composition granulométrique des trois stations de référence (AK8A, AK8B et AK8C) est quant à elle restée constante entre mai 2012 et mars 2013, avec des pourcentages en sables fins supérieurs à 48\% (station AK8B, novembre 2012, figure 3) et une fraction de vase inférieure à $1 \%$.

Les enregistrements vidéo réalisés à l'aide d'un mini ROV Seabotix ont permis d'apporter des informations complémentaires montrant notamment l'hétérogénéité des fonds. Avant le début des immersions, les fonds de la zone de Machu étaient caractérisés par des rides sableuses de courant (figures 4A et 4B). Durant les immersions, une importante hétérogénéité des fonds a pu être observée sur les sites de clapage. Des galets, des blocs de vase ou encore des dépôts vaseux indurés apparaissent sous forme de "plaques" (figures 4C, 4D, 4E et 4F). D'importants déplacements de sables autochtones viennent ensuite recouvrir les matériaux de dragage, sous l'action des forts courants de marée régnant dans ce secteur (AVOINE et al., 1986). Les vidéos et les photographies soulignent par conséquent l'influence locale des clapages sur les stations de dépôt direct et sur les stations voisines, illustrant les résultats des analyses granulométriques ainsi que ceux des levés bathymétriques.

\section{Conclusions}

Les 12 suivis réalisés pendant les clapages expérimentaux ont permis d'évaluer les changements des caractéristiques morpho-sédimentaires sur les deux sites d'immersion, MABIO (dépôts étalés) et MASED (dépôts concentrés), et à leur voisinage. L'impact des immersions a été immédiat, mis en évidence par des variations spatio-temporelles significatives des paramètres étudiés. Néanmoins, les effets ont été localisés et modérés aux zones de clapage et sur les zones les plus proches $( \pm 500 \mathrm{~m})$ comme le souligne la proportion de particules les plus fines (vase). De plus, les photographies et vidéos sous- 


\section{XIII ${ }^{\text {èmes }}$ Journées Nationales Génie Côtier - Génie Civil \\ Dunkerque, 2-4 juillet 2014}

marines soulignent une remarquable hétérogénéité des fonds dans le secteur de Machu suite aux immersions. La texture du sédiment qui est reconnu comme le facteur structurant fortement les communautés benthiques peut avoir un effet direct sur la distribution spatio-temporelle de la macrofaune benthique, comme les dépôts épais qui peuvent provoquer l'étouffement ou l'écrasement des organismes.

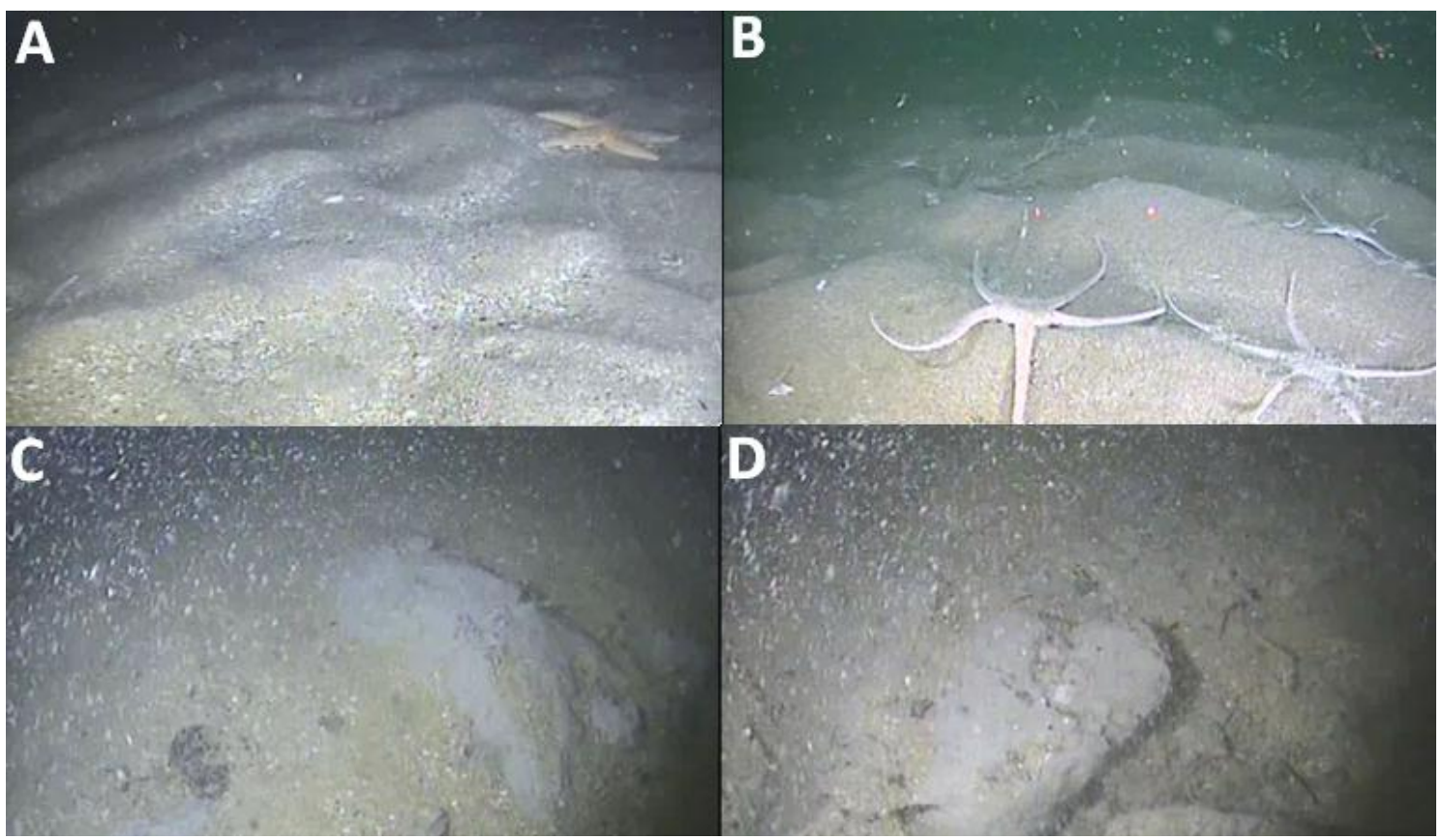

Figure 4. Exemples de clichés des fonds : (A) AK4D en novembre 2011 : rides de sables fins ; (B) AK8B en novembre 2011 : rides de sables fins et ophiures ; (C) AK4D en juin 2012 : morceaux de vase en partie recouverts de sables coquilliers ; (D) AK7D en juin 2012 : morceau de vase dans des sables coquilliers.

\section{Remerciements}

Ce travail a été réalisé dans le cadre d'une bourse CIFRE financée par le Grand Port Maritime de Rouen. Les auteurs remercient les équipages des navires Côtes de la Manche et Celtic Warrior ainsi que l'ensemble des personnes qui ont participé aux campagnes de prélèvement pour leur aide précieuse. Le personnel du Service du Chenal et à l'Aide à la Navigation (SCAN) du GPMR est aussi remercié pour l'étude bathymétrique ainsi que Carole Brunaud et Coralie Thouroude du laboratoire M2C de Caen pour les analyses granulométriques. 
Thème 6 - Gestion durable des zones littorales et estuariennes

\section{Références bibliographiques}

ALIZIER S., DAUVIN J.C. (2009). Résultats sur la campagne d'échantillonnage benthique en avril 2009 en baie de Seine. Station Marine de Wimereux, Contrat USTL ROCVN 9019, 39 p.

AVOINE J., CAILLOT A., HOSLIN R., LARSONNEUR C., MASSIAS J., QUESNEY M. (1986). Contribution des traceurs radioactifs à l'étude des mouvements sédimentaires en baie de Seine. La baie de Seine (GRECO-MANCHE). IFREMER, Université de Caen, pp 211-218.

BOLAM S.G., REES, H.L., SOMERFIELD P., SMITH R., CLARKE K.R., WARWICK, R.M., ATKINS M., GARNACHO E. (2006). Ecological consequences of dredged material disposal in the marine environment: A holistic assessment of activities around the England and Wales coastline. Marine Pollution Bulletin, Vol. 52, pp 415-426. http://dx.doi.org/10.1016/j.marpolbul.2005.09.028

FOLK R.L. (1966). A review of grain-size parameters. Sedimentology, Vol. 6, pp 73-93. http://dx.doi.org/10.1111/j.1365-3091.1966.tb01572.x

FOLK R.L., WARD W.C. (1957). Brazos River bar: a study in the signifiance of grain size parameters. Journal of Sedimentary Petrology, Vol. 27, pp 3-26. http://dx.doi.org/10.1306/74D70646-2B21-11D7-8648000102C1865D

HARVEY M., GAUTHIER D., MUNRO J. (1998). Temporal changes in the composition and abundance of the macrobenthic invertebrate communities at dredged material disposal sites in the Anse à Beaufils, baie des Chaleurs, eastern Canada. Marine Pollution Bulletin, Vol. 36, pp 41-55. http://dx.doi.org/10.1016/S0025-326X(98)90031-5 JOURDE J., DUBUT S. (2009). Etat initial biosédimentaire sur deux sites alternatifs d'immersion du Grand Port Maritime de Rouen : Machu et Banc de Seine. Rapport de la campagne de décembre 2008. Cellule du Suivi du Littoral Normand, 14 pp

MARMIN S. (2013). Impacts biosédimentaires des expérimentations de clapages en baie de Seine sur la communauté des sables moyens propres à Nephtys cirrosa. Thèse de doctorat, Université de Caen Basse Normandie, 249 p.

SIMONINI R., ANSALONI I., CAVALLINI F., GRAZIOSI F., IOTTI M., MASSAMBA N'SIALA G., MAURI M., MONTANARI G., PRETTI M., PREVEDELLI D. (2005). Effects of long-term dumping of dredged material on macrozoobenthos at four disposal sites along the Emilia-Romagna coast (Northern Adriatic Sea, Italy). Marine Pollution Bulletin, Vol. 50, pp 1595-1605. http://dx.doi.org/10.1016/j.marpolbul.2005.06.031

WENTWORTH C.K. (1922). A scale of grade and class terms for clastic sediments. Journal of Geology, Vol. 30, pp 377-392. http://dx.doi.org/10.1086/622910 